\title{
A new formula for the saddle-to-scission time
}

\author{
David Boilley ${ }^{1}$, Anthony Marchix ${ }^{1}$, Beatriz $\mathrm{Jurado}^{2}$, and Karl-Heinz Schmidt ${ }^{3}$ \\ ${ }^{1}$ Grand Accélérateur National d'Ions Lourds (GANIL), CEA/DSM-CNRS/IN2P3, BP 55027, F-14076 Caen cedex 5, France \\ 2 CENBG, CNRS/ IN2P3, Univ. Bordeaux I, Le Haut Vigneau, B.P. 120, 33175 Gradignan, France \\ 3 GSI, Planckstraße 1, D-64291 Darmstadt, Germany
}

May 25, 2007

\begin{abstract}
We propose a new formula for the saddle-to-scission time that is more general that the one based on Kramers' approach. Its validity and applicability is then studied in detail. Such a formula is useful for the evaluation of the fission time of very heavy nuclei.
\end{abstract}

PACS. 02.50.Ey Stochastic processes - 05.10.Gg Stochastic analysis methods (Fokker-Planck, Langevin, etc.) - 24.75.+i General properties of fission - 25.70.Jj Fusion and fusion-fission reactions

\section{Introduction}

Kramers formula [1] for the stationary escape rate from a metastable well under friction in a thermal environment, proposed one year after the discovery of nuclear fission, is still used nowadays in the case of thermal fission. Besides its simplicity, the fact that the fission rate is defined by a master equation for the compound nucleus population $P_{C N}$,

$$
d P_{C N}=-\Gamma_{f} P_{C N} d t
$$

allows one to introduce this decay channel into a more general cascade scheme, which, when integrated out, gives back the statistical model $[2,3]$. This may be the main reason for its success, since it is well known that the fis- sion time is determined by the competition of the fission channel with the evaporation of light particles and $\gamma$-ray emission. Unfortunately, the domain of application of the escape-rate concept is limited. In addition, to get the formula itself, some approximations are needed.

This is particularly the case for the evaluation of the fission time. The escape rate defined by Kramers gives access to the escape-time distribution at the saddle point. For very heavy nuclei, the time necessary to go from the saddle to the scission point cannot be neglected. The Mean First Passage time (MFPT), for which a simple formula is available in the high friction limit $[4,5]$, could be a useful theoretical tool. Unfortunately, it is only an average 
time and we would rather need a first passage time distribution to consider the evaporation of light particles in a statistical-model simulation. For this purpose, one generally keeps Kramers' formalism and adds a saddle-toscission time [6]. A simple formula was proposed in Refs [7, 8], based on the hypothesis used by Kramers: a parabolic potential and low temperature. In this paper, we propose a more general formula for the saddle-to-scission time that goes beyond Kramers' approximation.

In addition, numerical simulations using either the KleinKramers equation [9] or the Langevin one [10] have shown that at high excitation energies the finite time needed to build up the quasi-stationary probability flow over the fission barrier is long enough to be comparable to the lifetimes for desintegration channels such as particle evaporation. This fact is now commonly accepted $[6,11,12]$ but a general analytical formula for the transient regime that depends on the initial conditions is so far not available. This important aspect goes beyond the scope of this paper, which is limited to the saddle-to-scission time.

\section{General derivation in the overdamped limit}

As already mentionned in the introduction, the MFPT is a useful tool to evaluate the time needed to reach the scission point. When the friction coefficient is large enough to neglect the inertia, the Klein-Kramers equation $[13,1]$ can be reduced to the Smoluchowski one [14] and an analytical formula is available for the MFPT $[4,5]$. Assuming a metastable potential well, $V(x)$, with an arbitrary but fixed initial condition in $x_{i}$ and an absorbing point at $x_{e}$ one has,

$$
\operatorname{MFPT}\left[x_{i} \rightarrow x_{e}\right]=\frac{m \beta}{T} \int_{x_{i}}^{x_{e}} d y e^{\frac{V(y)}{T}} \int_{-\infty}^{y} d z e^{-\frac{V(z)}{T}}
$$

Here, $\beta$ is the reduced friction and $T$, the temperature. As usual in nuclear physics, the Boltzmann constant is set to

1. The boundary conditions of the MFPT are well adapted to the fission problem if the exit point $x_{e}$ is chosen at the scission point.

But at the saddle the situation differs. The MFPT calculation supposes that a sink absorbs all the coming particles whereas the Kramers' rate includes backward currents due to particles that can cross several times over the barrier. The stationary positive and negative currents over the barrier, defined as,

$$
\begin{aligned}
& j_{+}(x)=\int_{0}^{+\infty} d v v W(x, v), \\
& j_{-}(x)=\int_{-\infty}^{0} d v v W(x, v),
\end{aligned}
$$

where $W(x, v)$ is the stationary probability distribution, can easily be evaluated in the same way as Kramers' stationary rate (see e.g. Ref. [15] for a detailed calculation of the latter). With the same approximations, one gets,

$$
\begin{aligned}
& j_{+}\left(x_{b}\right)=\frac{j_{K}}{2}\left[1+\frac{\beta}{2 \omega_{b}}+\sqrt{1+\left(\frac{\beta}{2 \omega_{b}}\right)^{2}}\right] \\
& j_{-}\left(x_{b}\right)=\frac{j_{K}}{2}\left[1-\frac{\beta}{2 \omega_{b}}-\sqrt{1+\left(\frac{\beta}{2 \omega_{b}}\right)^{2}}\right]
\end{aligned}
$$

where $j_{K}$ is Kramers' stationary current. Here, $\omega_{b}$ characterises the osculatory parabola at the barrier. For very large viscosities $\left(\beta \gg 2 \omega_{b}\right)$, the previous results become,

$$
\begin{aligned}
& j_{+}\left(x_{b}\right) \simeq j_{K}\left(\frac{1}{2}+\frac{\beta}{2 \omega_{b}}+\frac{\omega_{b}}{2 \beta}\right), \\
& j_{-}\left(x_{b}\right) \simeq j_{K}\left(\frac{1}{2}-\frac{\beta}{2 \omega_{b}}-\frac{\omega_{b}}{2 \beta}\right) .
\end{aligned}
$$


Both positive and negative currents are larger than the Kramers current which results from the difference between them. Beyond the saddle, assuming that locally the potential is an inverted parabola, the backward current vanishes when $V\left(x_{b}\right)-V\left(x_{e}\right) \gg T$. Similar results were obtained in Ref. [16].

At the saddle, to cope with the negative currents, one should rather define a Mean Passage Time (MPT). Calling $P\left(t, x_{b} ; x_{i}\right)$ the probability that a particle launched at $t=$ 0 in $x_{i}$ situated in the potential well is still in the well at time $t$, the escape current at the barrier top $x_{b}$, defined as

$$
j\left(t, x_{b} ; x_{i}\right)=-\frac{\partial P\left(t, x_{b} ; x_{i}\right)}{\partial t}
$$

gives the distribution of the escape times. Eq. (9) is a consequence of the continuity equation. Then, the MPT could simply be evaluated,

$$
\begin{aligned}
\operatorname{MPT}\left[x_{i} \rightarrow x_{b}\right] & =\int_{0}^{+\infty} t j\left(t, x_{b} ; x_{i}\right) d t \\
& =\int_{0}^{+\infty} P\left(t, x_{b} ; x_{i}\right) d t .
\end{aligned}
$$

The second line was obtained by a trivial integration by part, using the fact that $P\left(t, x_{b} ; x_{i}\right)$ vanishes for large times. The Mean Passage Time coincides with the Non Linear Relaxation Time (NLRT) [17] defined by eq. (11) when $P\left(t=0, x_{b} ; x_{i}\right)=1$ and $P\left(t \rightarrow \infty, x_{b} ; x_{i}\right)=0$.

In Ref. [18], analytical formulas are derived for the NLRT for various kinds of potentials from the Smoluchowski equation. For the metastable well we are interested in here, one has,

$$
\begin{aligned}
N L R T\left[x_{i} \rightarrow x_{e}\right] & =\frac{m \beta}{T}\left[\int_{x_{i}}^{x_{e}} d y e^{\frac{V(y)}{T}} \int_{-\infty}^{y} d z e^{-\frac{V(z)}{T}}\right. \\
& \left.+\int_{x_{e}}^{\lambda} d y e^{\frac{V(y)}{T}} \int_{-\infty}^{x_{e}} d z e^{-\frac{V(z)}{T}}\right],
\end{aligned}
$$

where $\lambda$ is an absorbing condition that could be at the scission point. If the exit point $x_{e}$ is chosen at the absorbing limit $\lambda$, this formula gives back the MFPT, eq. (2).

In Ref. [19], some universal relationships between these escape times were derived. The main results are recalled here. The MFPT needs, by definition, an absorbing point at the boundary and is therefore only suitable to evaluate the average time necessary to reach the scission point. This constraint does not exist with the NLRT which can be used at the saddle. The difference between these two times is called the saddle-to-scission time:

$$
\tau_{b \rightarrow s}=\operatorname{MFPT}\left[x_{i} \rightarrow x_{s}\right]-N L R T\left[x_{i} \rightarrow x_{b}\right] .
$$

The NLRT itself includes the long-time-limit escape rate, $\Gamma_{\infty}$ well approximated by Kramers' formula, and the transient time. Unfortunately, no general formula is available to link these quantities, except in the very specific case where the transient function is approximated by a step function up to the time $\tau_{r}$,

$$
\Gamma(t)=\Theta\left(t-\tau_{r}\right) \Gamma_{\infty}
$$

where one gets

$$
N L R T\left[x_{i} \rightarrow x_{b}\right]=\tau_{r}+\frac{1}{\Gamma_{\infty}}
$$

A clear distinction should be done between the NLRT and the MFPT that are average times and the escape rate approach which gives access to the escape-time distribution, see eq. (1). It is then natural that both the NLRT and the MFPT include the transient time. Such a result was already shown in Ref. [20] in a different context, but contradicts the main conclusion of Ref. [21]. 
From the expressions of the MFPT and the NLRT, Eqs (2) and (12) respectively, it is easy to get a simple formula for the saddle-to-scission time that has the merit of being exact for very large friction:

$$
\tau_{b \rightarrow s}=\frac{m \beta}{T} \int_{x_{b}}^{x_{s}} d y e^{\frac{V(y)}{T}} \int_{x_{b}}^{y} d z e^{-\frac{V(z)}{T}} .
$$

Such a formula is also valid for potentials having a structure beyond the saddle point.

\section{Extension to medium friction}

Although there is still a large uncertainty on the viscosity of nuclear matter at finite temperature, the approximation used for the general formula obtained in the previous subsection is probably too crude in the nuclear context and has to be extended to medium viscosities.

Kramers' stationary escape rate, which can be applied down to medium viscosities, was shown to correspond to the escape rate obtained in the transition-state theory corrected by a prefactor characterising the dynamics of the barrier [22]. Both the MFPT and the NLRT formulas, Eqs. (2) and (12) have a similar structure. Therefore, we suggest replacing the prefactor in these formulas by chang$\operatorname{ing} \beta$ :

$$
\beta \rightarrow \frac{1}{2}\left(\beta+\sqrt{\beta^{2}+4 \omega_{b}^{2}}\right) .
$$

Such a prefactor is similar to the one obtained in Refs $[7,8]$ with a derivation based on Kramers' approach [1]. It comes from the study of the dynamics of a parabolic barrier $[23,24]$.

Note that this correction is based on the hypothesis that there is a single barrier. The study of potentials hav-
Fig. 1. Dimensionless plots for the $N L R T\left[x_{i}=0 \rightarrow x_{b}\right]$ at the barrier $x_{b}$ as a function of the scission point $x_{s}$ evaluated numerically and analytically with the exact formula in the overdamped regime and the corrected one. $x=0$ corresponds to the bottom of the well. Figures a) and b) are calculated for a quadratic potential, with a medium friction $\frac{\beta}{2 \omega}=2$ and at two temperatures, $\frac{T}{B}=0,5$ and $\frac{T}{B}=2$ respectively. Figure c) is calculated with a very large friction $\frac{\beta}{2 \omega}=10$ for a quadratic potential at temperature $\frac{T}{B}=2$. Figure d) is calculated for a cubic potential with a medium friction $\frac{\beta}{2 \omega}=2$ at a large temperature $\frac{T}{B}=2$.

ing a structure beyond the saddle is out of the scope of this paper.

This correction was checked numerically with a Langevin equation [25] for various potentials, temperatures and viscosities. In Fig. 1, a typical example is shown for the NLRT. For a medium friction coefficient, $\frac{\beta}{2 \omega}=2$, the corrected formula differs quite a lot from the one derived in the high-friction limit and is in good agreement with the numerical evaluation. See Figs. 1a and 1b. When the scission point is close to the saddle, i.e. when $V\left(x_{b}\right)-V\left(x_{s}\right)<$ $T$, the disagreement is partly due to the statistical fluctuation of the numerical simulation. In the overdamped regime, the NLRT formula is exact and we can still observe the same discrepancies, see Fig. 1c. With a cubic potential, Fig 1d, the agreement limit, $V\left(x_{s}\right) \simeq V\left(x_{b}\right)-T$ is reached for smaller values of $x_{s}$.

The results are similar for the MFPT and then for the saddle-to-scission time. 
Fig. 2. Dimensionless plot comparing three formulas for the saddle-to-scission time. The solid curve corresponds to the formula of Refs. $[7,8]$, the dotted one to the new formula, Eq. (19), and the dashed one to the approximate formula, Eq. (21).

\section{Comparison with other formulas}

With a potential landscape assumed to be parabolic between the saddle and scission points,

$$
V(x)=B-\frac{1}{2} m \omega_{b}^{2}\left(x-x_{b}\right)^{2}
$$

the saddle-to-scission time simply becomes,

$\tau_{b \rightarrow s}=\frac{\sqrt{\beta^{2}+4 \omega_{b}^{2}}+\beta}{\omega_{b}^{2}} \int_{0}^{\sqrt{\frac{m}{2 T}} \omega_{b}\left(x_{s}-x_{b}\right)} d y e^{-y^{2}} \int_{0}^{y} d z e^{z^{2}}$

and can be compared to the result of Refs. [7,8]. A comparison is shown on Fig. 2 for a very large friction for which our formula is exact. The behavior for weaker friction is similar.

When the scission point is far away from the saddle point, the two formulas coincide nicely. It is not the case when the scission point is close to the saddle. This is due to the fact that in Refs. $[7,8]$ the evaluation is based on the current and then includes an up stream contribution, whereas in our case, it is based on the MFPT formalism which assumes an absorbing boundary which is better adapted to the context of nuclear fission.

The difference of the behavior can be easily understood if we consider that the potential is almost flat near the barrier. Then, we can reverse Einstein's free diffusion formula to get, with a reflecting point in $x_{i},[26,5]$,

$$
\operatorname{MFPT}\left[x_{i} \rightarrow x_{e}\right]=\frac{m \beta}{2 T}\left(x_{e}-x_{i}\right)^{2}
$$

Fig. 3. Relative error between the approximate formula for the saddle-to-scission and the new formula defined by Eq. (22).

Fig. 2 shows that the new formula leads to a parabolic behavior near the origin where the formula of Refs. $[7,8]$ is linear.

Eq. (19) is not so easy to compute and takes time to evaluate in a cascade code. We propose then an approximate formula only valid for a parabolic potential between the saddle and scission points:

$$
\tau_{b \rightarrow s} \simeq \frac{\sqrt{\beta^{2}+4 \omega_{b}^{2}}+\beta}{2 \omega_{b}^{2}} \log \left(2.7 \sqrt{\frac{V\left(x_{b}\right)-V\left(x_{s}\right)}{T}}-0.3\right) .
$$

Fig. 2 shows that it fits well Eq. (19), far enough from the saddle. For practical reasons, it is useful to estimate the accuracy of this approximate expression. In Fig. 3, we plot the relative error defined as

$$
\frac{\tau_{b \rightarrow s}^{\text {approx }}-\tau_{b \rightarrow s}}{\tau_{b \rightarrow s}}
$$

where $\tau_{b \rightarrow s}^{\text {approx }}$ is given by Eq. (21) and $\tau_{b \rightarrow s}$ by Eq. (19). Note that this relative error does not depend on the reduced friction $\beta$.

\section{Role of the potential}

One has to be cautious with the application of this formula for higher order potentials.

For a cubic potential in the high-friction limit, the deterministic equation of motion can be evaluated analytically:

$$
x(t)=\frac{x_{0} x_{i} \exp \left(\omega_{b}^{2} t / \beta\right)}{x_{0}+x_{i}\left(\exp \left(\omega_{b}^{2} t / \beta\right)-1\right)},
$$


where $x_{i}$ corresponds to the initial condition. To get this expression, the general cubic potential is written in a convenient way,

$$
V(x)=m \omega_{b}^{2}\left(-\frac{x^{2}}{2}+\frac{x^{3}}{3 x_{0}}\right),
$$

where $x_{0}<0$ refers to the bottom of the well. The osculatory parabolic potentials in the well and at the saddle (in $x=0$ ) have a pulsation equal to $\omega_{b}$. When $x_{i}$ is outside the saddle $\left(x_{i}>0\right)$, it takes a finite time $\left(t_{\infty}=\right.$ $\left.\beta \ln \left(1-x_{0} / x_{i}\right) / \omega_{b}^{2}\right)$ for $x(t)$ to reach infinity. Such a behavior is also observed in the numerical solution of the full deterministic equation of motion where the inertia is not neglected. Since the stochastic equations are not linear, the previous results do not represent the average value, but one finds similar results with a Langevin equation. Then, the observation that the MFPT does not depend on $x_{e}$ is simply due to the strength of the potential, for which the force increases without limit as $x$ increases, leading to unphysical results.

The saturation for large $x_{e}$ observed in Ref. [27] for higher power potentials occurs for the same reason. Starting the calculation at $x_{i}$ far away from the saddle, the potential reduces to $V(x) \simeq-c . x^{n}$. Then, the time to reach infinity is given by

$$
t_{\infty}=\int_{x_{i}}^{\infty}-\frac{m \beta}{d V / d x} d x=\frac{m \beta}{n(n-2) c x_{i}^{n-2}},
$$

for $n>2$, which is also finite.

With a parabolic potential, it is not the case. Outside the saddle, an analytic expression for the average trajectory is available in Ref. [23]. For large times,

$$
\langle x(t)\rangle \propto \exp (a t) \quad \text { with } \quad a=\frac{1}{2}\left(\sqrt{\beta^{2}+4 \omega_{b}^{2}}-\beta\right) .
$$

Then, the MFPT should increase proportionally to $\ln \left(x_{e}\right) / a$ for large $x_{e}$. In the overdamped limit, $a \simeq \omega_{b}^{2} / \beta$. This result could also be obtained from the MFPT formula, eq. (2). For large $x_{e}$, one has,

$$
\begin{aligned}
\frac{\partial M F P T\left[x_{i} \rightarrow x_{e}\right]}{\partial x_{e}} & \simeq \frac{m \beta}{T} e^{-\frac{m \omega_{b}^{2} x_{e}^{2}}{2 T}} \int_{0}^{x_{e}} d v e^{\frac{m \omega_{b}^{2} v^{2}}{2 T}},(27) \\
& \rightarrow \frac{\beta}{\omega_{b}^{2} x_{e}} .
\end{aligned}
$$

With a cubic potential, numerical calculations show that the previous derivative vanishes for large $x_{e}$.

This analysis shows that we have to be cautious in the application of these formulas when the scission point is far from the saddle one. The same problem would arise with a numerical simulation based on classical mechanics.

\section{Role of memory effects}

The present study is based on a Markovian approximation, assuming that the heat bath relaxes faster than the charateristic time of the collective variable. When the Langevin equation is derived from a microscopic model, one obtains a memory kernel which is due to the coupling of the collective variable to the deformation of the Fermi surface $[28-30]$.

For a typical kernel, an exact solution of the nonMarkovian evolution over a parabolic barrier is given in Ref. [31]. In this reference, the reduced friction coefficient and the relaxation time of the heat bath are artificially considered as independent parameters. It is shown that, for a given friction parameter, non-Markovian dynamics lead to diminish the effect of the viscosity and then to a shorter saddle-to-scission time. In addition, the memory 
kernel gives rise to oscillations along the drift path to scission at the frequency of the giant quadrupole resonance that may break up the nucleus earlier.

But when the friction parameter is derived from a microscopic model [28-30], it depends on the relaxation time chosen for the memory kernel. In $[29,30] \beta$ is proportionnal to this relaxation time. Then, a larger relaxation time also means a larger viscosity with a net effect of a longer saddle-to-scission time. This last tendency was also pointed in Ref. [28] for another memory kernel, where the authors give an estimate: "due to the memory effects the saddle-to-scission time grows by a factor of about 3 with respect to the corresponding saddle-to-scission time obtained in liquid drop model calculations with friction forces".

Our new formula does not include any memory effect.

\section{Conclusion}

In a multi-channel de-excitation scheme the fission process competes with other decay modes. If a decay happens in another channel before the saddle point is reached, the subsequent decrease of the available energy will greatly hinder fission. Therefore, one generally takes into account the fission process up to the saddle only. But, for very heavy nuclei the scission point is very far from the saddle and one cannot neglect the saddle-to-scission time. In this paper we propose a new formula to evaluate such a time that is exact in the high-dissipation limit. Since the nuclear viscosity may not be that large, we propose a correction to the formula that has been checked numerically.
We showed that a high order potential leads to unphysical results far from the saddle. The applicability of a classical saddle-to-scission dynamics is then limited. This explains why we can apply locally a parabolic approximation to the potential in order to improve the formula of the saddle-to-scission time.

\section{References}

1. H. A. Kramers, Physica VII, 4, 284 (1940)

2. S. Hassani and P. Grangé, Phys. Lett. B137, 281 (1984)

3. B. Bouriquet, Y. Abe and D. Boilley, Comp. Phys. Comm. $\mathbf{1 5 9 , 1}(2004)$

4. L. Pontryagin, A. Andronov and A. Vitt, Zh. Eksp. Teor. Fiz. 3, 165 (1933); translated in English in Noise in Nonlinear dynamics ed. by F. Moll and P.V.E. McClintock, Cambridge University Press, Vol. 1, p. 329 (1989)

5. G. Klein, Proc. R. Soc. London A211, 431 (1952)

6. D. Hilscher and H. Roßner, Ann. Phys. Fr. 17, 471 (1992)

7. H. Hofmann and J.R. Nix, Phys. Lett. 122B, 117 (1983)

8. J.R. Nix, A.J. Sierk, H. Hofmann, F. Scheuter and D. Vautherin, Nucl. Phys. A424, 239 (1984)

9. P. Grangé, J.-Q. Li and H.A. Weidenmüller, Phys. Rev. C27, 2063 (1983)

10. Y. Abe, C. Grégoire and H. Delagrange, J. de Phys. C4, 329 (1986)

11. Y. Abe, S. Ayik, P.-G. Reinhard and E. Suraud, Phys. Rep. 275, 49 (1996)

12. P. Fröbrich and I.I. Gontchar, Phys. Rep. 292, 131 (1998)

13. O. Klein, Arkiv. Mat. Astr. Fys. 16, 1 (1921)

14. M. von Smoluchowski, Ann. der Phys. (Leipzig) 48, 1103 (1915) 
15. P. Hänggi, P. Talkner and M. Borkovec, Rev. Mod. Phys. 62, $251(1990)$

16. J.-D. Bao and Y. Jia, Phys. Rev. C69, 027602 (2004)

17. M. Suzuki, Int. J. Magn. 1, 123 (1971)

18. A. N. Malakhov, Chaos 7, 488 (1997)

19. D. Boilley, B. Jurado and C. Schmitt, Phys. Rev. E70, $056129(2004)$

20. M. Bier, I. Derényi, M. Kostur and R.D. Astumian, Phys. Rev. E59, 6422 (1999)

21. H. Hofmann and F. A. Ivanyuk, Phys. Rev. Lett. 90, $132701(2003)$

22. D. Kohen and D.J. Tannor, J. Chem. Phys. 100, 4932 (1994)

23. Y. Abe, D. Boilley, B.G. Giraud and T. Wada, Phys. Rev. E61, 1125 (2000)

24. D. Boilley, Y. Abe and J.D. Bao, Eur. Phys. J. A18, 627 (2003)

25. P. Langevin, Comptes Rendus de l'Acad. des Sciences Paris, 146, 530 (1908)

26. R. Fürth, Ann. der Phys. (Leipzig) 53, 177 (1917)

27. H. Hofmann and A.G. Magner, Phys. Rev. C68, 014606 $(2003)$

28. V.M. Kolomietz, S.V. Radionov and S. Shlomo, Phys. Rev. C64, $054302(2001)$

29. S. Ayik, E. Suraud, J. Stryjewski and M. Belkacem, Z. Phys. A 337, 413 (1990)

30. D. Boilley, Y. Abe, S. Ayik and E. Suraud, Z. Phys A 349, 119 (1994)

31. D. Boilley and Y. Lallouet, J. Stat. Phys. 125, 477 (2006) 

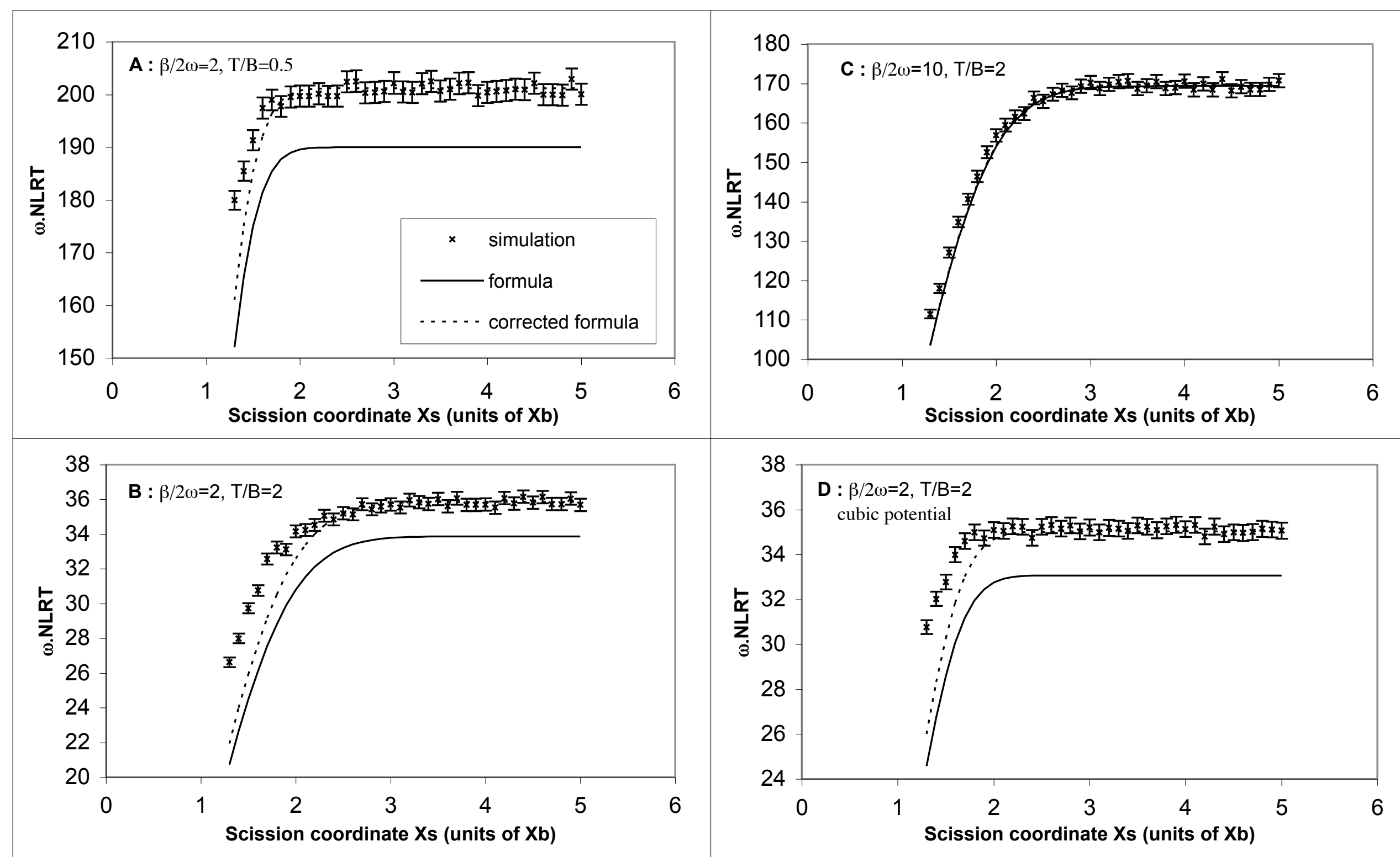


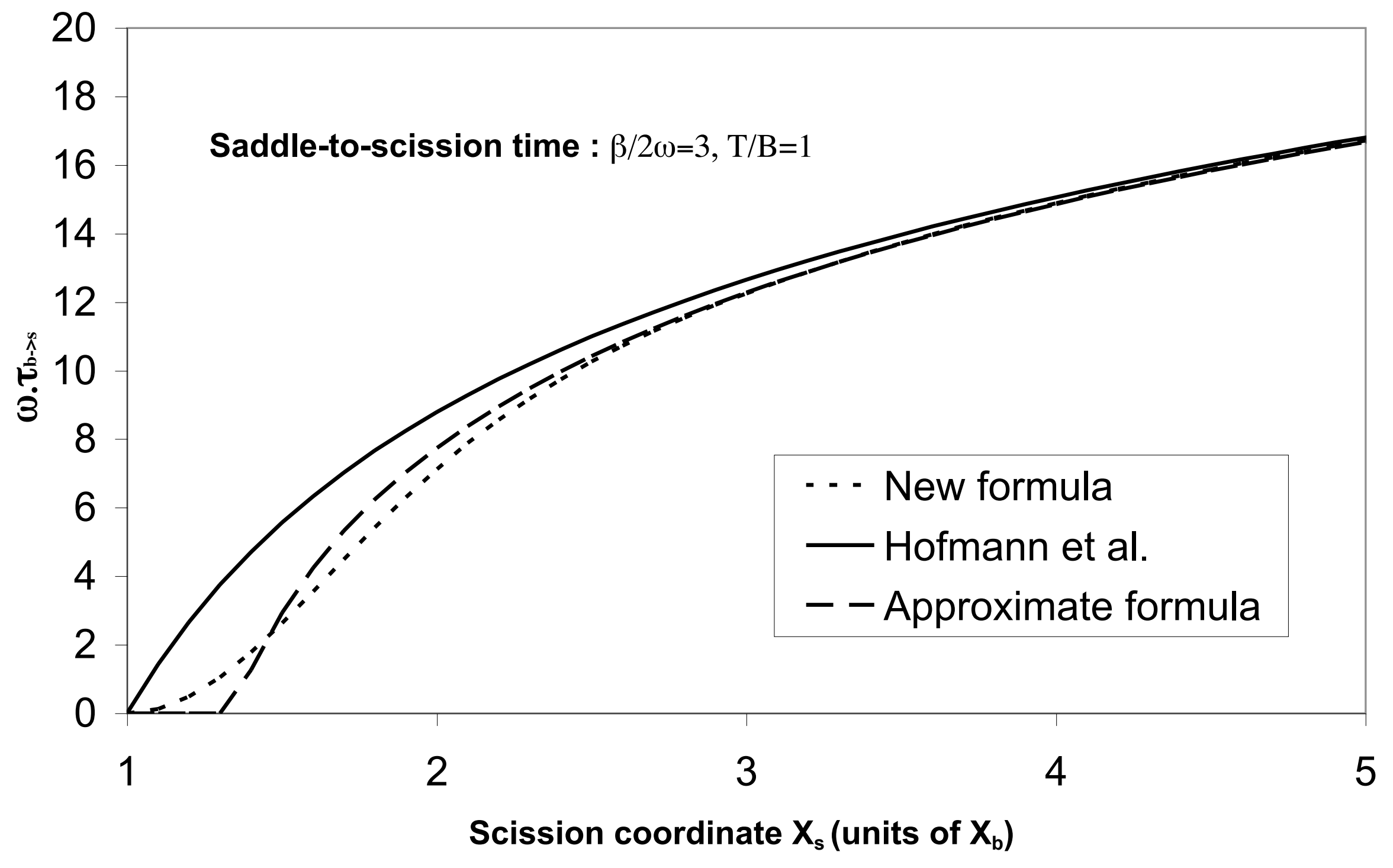




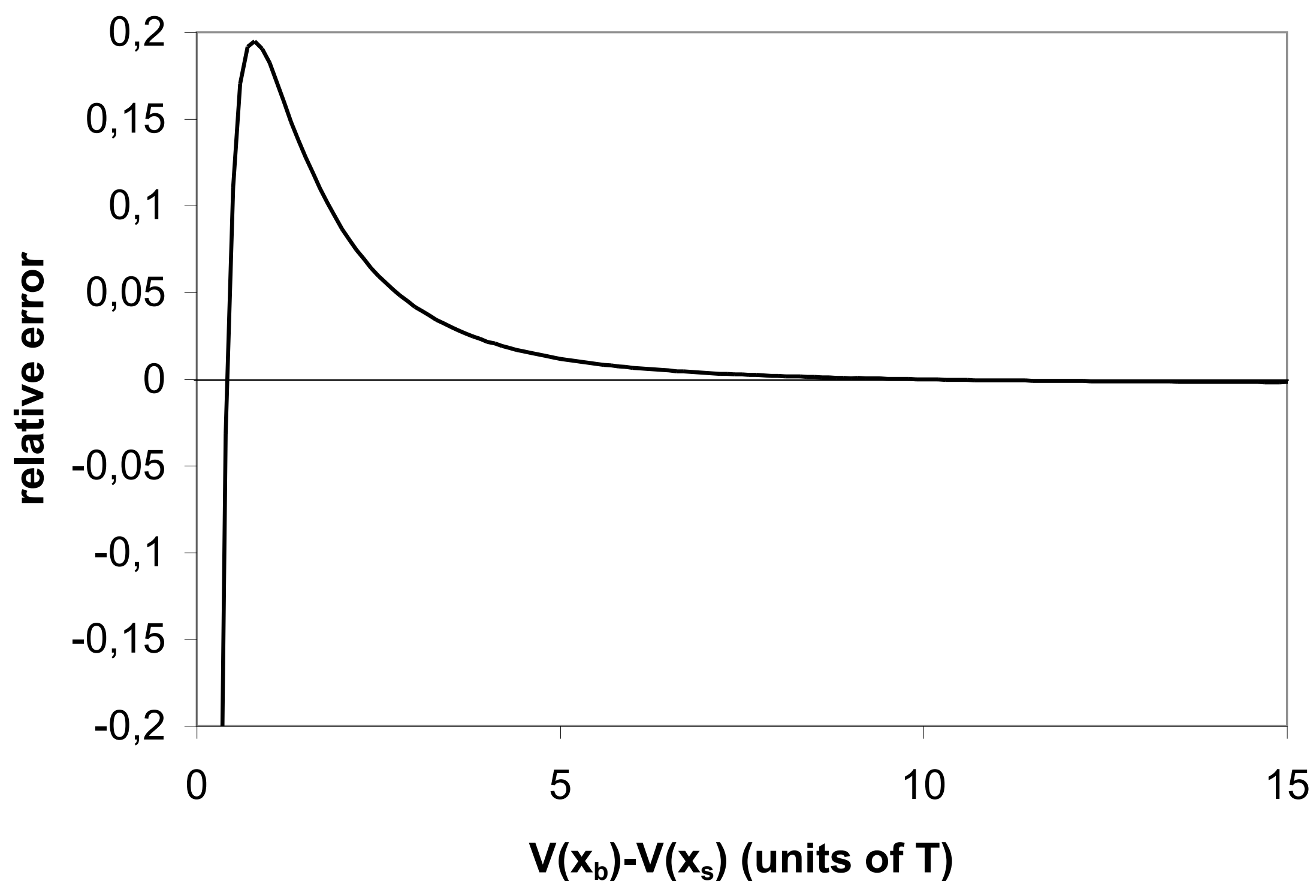

